\title{
Micro Electron Diffraction is a quick and versatile tool for structure determination of macromolecules and small molecules
}

\author{
Alevtyna Yakushevska ${ }^{1}$, Steve Reyntjens ${ }^{1}$, \\ ${ }^{1}$ Materials and Structural Anlysis Division, Thermo Fisher Scientific, Eindhoven, \\ The Netherlands. \\ alevtyna.yakushevska@thermofisher.com
}

X-ray crystallography experiments require large, well-ordered protein crystals for diffraction data collection. Growing protein crystals is difficult, requires a lot of time and effort and it is sometimes even impossible. However, crystallization experiments quite often produce plenty of microcrystals which are too small for conventional X-ray diffraction experiments. Moreover, large protein crystals are frequently imperfect and often suffer from different defects like high mosaicity. Small crystals are usually not affected by such defects and may yield better quality data. These kind of small crystals could be used for high-resolution structure determination by electron microscopy methods.

Cryo-transmission electron microscope (cryo-TEM) and diffraction are increasingly powerful methods for the analysis of biological structures at near atomic resolution. However, the very strong interaction of electrons with matter limits the thickness of specimens that can be analyzed at high resolution, by the current implementations of these methods, to a few hundreds of nanometers. To efficiently collect MicroED data, software automation, dedicated hardware components and customized optical setting can be installed on a cryo-TEM. Combined with the intrinsic microscope performance, we show that data collection is now fully automated and can be realized in a matter of minutes. 